\title{
APOE is a potential modifier gene in an autosomal dominant form of frontotemporal dementia (IBMPFD)
}

Sarju G. Mehta, MD ${ }^{1,5}$, Giles DJ. Watts ${ }^{1,5}$, Jennifer L. Adamson ${ }^{2}$, Mike Hutton ${ }^{2}$, Geanie Umberger, PhD ${ }^{3}$, Shuling Xiong, $\mathrm{MD}^{4}$, Sheena Ramdeen, $\mathrm{BSc}^{1}$, Mark A. Lovell, $\mathrm{PhD}^{4}$, Virginia E. Kimonis, $\mathrm{MD}^{1}$, and Charles D. Smith, $M D^{3}$

\begin{abstract}
Purpose: Inclusion-body myopathy, Paget's disease of bone and frontotemporal dementia is an adult-onset autosomal dominant illness (IBMPFD) caused by mutations in the valosin-containing protein (VCP) on chromosome 9p21.1-p12. The penetrance of the gene is $82 \%$ for myopathy, $49 \%$ for Paget's disease, but may be as low as 30\% for frontotemporal dementia. Modifier genes could account for decreased frontotemporal dementia penetrance. In this study apolipoprotein-E ( $A P O E$ ) was evaluated for this role in IBMPFD families based on its known modifier effect in Alzheimer's disease. Methods: From a database of 231 members of 15 families, 174 had APOE genotype available for analysis. Logistic regressions on $A P O E$ genotype and frontotemporal dementia were performed, using appropriate covariates. Results and Conclusion: FTD was associated with $A P O E 4$ genotype $(P=0.0002)$, myopathy $(P=0.0006)$, and age $(P=0.01)$, but not microtubule associated protein tau (MAPT) $\mathrm{H} 2$ haplotype $(P=0.5)$ or gender (0.09) after adjustment for membership in pedigrees with at least one APOE 4 genotype. These data suggest a potential link between $A P O E 4$ genotype and the specific form of frontotemporal dementia found in IBMPFD. The molecular basis of this link bears further investigation. We did not observe an association of frontotemporal dementia and H2 MAPT haplotype. Genet Med 2007:9(1):9-13.
\end{abstract}

Key Words: Frontotemporal dementia, Paget's disease of bone, inclusion body myopathy, muscular dystrophy, apolipoprotein-E, valosin-containing protein

We previously reported a novel adult-onset autosomal dominant genetic disorder characterized by the cardinal features frontotemporal dementia, Paget's disease of bone, and inclusion body myopathy ${ }^{1}$ (IBMPFD; OMIM \#605382). Subsequently we located causative mutations in the same gene residing on chromosome 9 in thirteen families. ${ }^{2}$ The gene codes for valosin-containing protein (VCP), an AAA protein (ATPase Associated with a variety of Activities). ${ }^{3} \mathrm{VCP}$ was previously unknown to be related to any of the separate disorders constituting the three cardinal clinical manifestations of IBMPFD. Therefore, further investigation into IBMPFD, despite its rarity, might provide important insights into the pathogenesis of more common forms of frontotemporal dementia, Paget's disease of bone and inclusion body myopathy.

\footnotetext{
From the ${ }^{1}$ Children's Hospital Clinical Genetics and Metabolism, Boston, Massachusetts; ${ }^{2}$ Mayo Clinic Jacksonville, Jacksonville, Florida; ${ }^{3}$ Department of Neurology and SandersBrown Center on Aging, University of Kentucky, Lexington, Kentucky; ${ }^{4}$ Department of Chemistry and Sanders-Brown Center on Aging, University of Kentucky, Lexington, Kentucky; ${ }^{5}$ These authors contributed equally.

Virginia E. Kimonis, MD, MRCP, Division of Genetics and Metabolism, Department of Pediatrics, University of California Irvine Medical Center, 101 The City Drive South, ZC4482,Orange,CA 92686.E-mail:vkimonis@uci.edu

Submitted for publication May 3, 2006.

Accepted for publication October 31, 2006.

Disclosure: The authors reported no conflict of interests.
}

DOI: 10.1097/GIM.0b013e31802d830d
An intriguing observation in IBMPFD has been the variability of expression of the frontotemporal dementia (FTD) phenotype. When present, the FTD syndrome typically consists of agitation, expressive dysphasia and apathy with personality change and relative sparing of memory. However, FTD affected only $30 \%$ of patients in our previous clinical study, with an average age of onset of 55 years. ${ }^{1,2}$ The majority of these patients also had pre-existing IBM and/or PDB. Since not all affected individuals survive to age 55 because of severe myopathy, early death in some subjects with IBM may have led to an underestimate of the FTD phenotype. ${ }^{2}$ Nonetheless we observed that the incidence of FTD in at least one family with IBMPFD was particularly high (63\% of affected individuals; 5/8 individuals) with an average age of onset of 53 years, suggestive of modifying factors predisposing to FTD.

We investigated $A P O E$ as a potential modifier gene to account for the observed variation in FTD expression in IBMPFD. The epsilon-4 allele of apolipoprotein-E (APOE 4) is known to be a risk factor for Alzheimer's disease $(\mathrm{AD})^{4,5}$ and it has been implicated in other dementias including diffuse Lewy body disease ${ }^{6}$ and vascular dementia. The status of APOE in sporadic frontotemporal lobar degeneration and other subtypes remains controversial. ${ }^{7-10}$ Microtubule associated protein tau (MAPT) haplotype was also investigated because of its 
described modifier effect in frontotemporal lobar degeneration, and possibly in $\mathrm{AD} .{ }^{11-14}$

\section{METHODS}

The study was reviewed and approved by the IRB and informed consent was obtained from all subjects. Methods of clinical and biochemical investigation have been reported previously. ${ }^{1}$ IBMPFD-related FTD was diagnosed if there was a history of significant slowly progressive behavioral change or language disorder with onset prior to the age of 65 years in an affected subject. Living subjects were personally interviewed by the study staff, but some deceased subjects were assigned an FTD diagnosis by formal family interview listing typical dementia symptoms.

We extracted peripheral blood DNA using the PureGene DNA isolation kit (Gentra Systems, Minneapolis, MN). Mutation analysis was undertaken using methods described by Watts et al. ${ }^{2}$ sequencing with an ABI 377 sequencer, using a dRhodamine terminator cycle sequencing kit (Applied BioSystems, Foster City, CA).

$A P O E$ genotyping was carried out using the method of Addya et al. ${ }^{15}$ Briefly, 6 to $8 \mathrm{~mL}$ of blood containing heparin as an anticoagulant were centrifuged at $5000 \times \mathrm{g}$ for 5 minutes. The plasma was carefully removed and $200 \mu \mathrm{L}$ of buffy coat (white blood cell layer) was isolated for use in DNA isolation using a QIAampDNA blood Mini Kit (Qiagen, Inc., Valencia, CA). Five microliters of DNA was used for PCR analysis using the following primers: forward 5' G TCC AAG GAG CTG CAG GC 3' reverse -5' GGC GCT CGC GGA TGG CGC TGA G 3'. Amplification was performed in a PTC- $100^{\mathrm{TM}}$ Programmable Thermal Controller (MJ Research, Inc., San Francisco, CA) using PCR cycles as described by Addya et al. ${ }^{15}$ Following amplification, 10 units Hha I (Invitrogen Corp., Carlsbad, CA) were added to $25 \mu \mathrm{L}$ of the PCR product and incubated for 2 hours at $37^{\circ} \mathrm{C}$. The digested DNA was then separated by electrophoresis in a $5 \%$ agarose gel ( $4 \%$ low melting and $1 \%$ regular agarose).

The tau haplotype was inferred as previously described by assessing for the presence or absence of a 238 base pair deletion in intron 9 of $M A P T,{ }^{16}$ with presence defining $\mathrm{H} 1$ and absence defining $\mathrm{H} 2$ allele. Genotyping was conducted blinded to the clinical status.

Our IBMPFD database consists of 231 members of 15 identified IBMPFD families enrolled in a longitudinal study of the disease. ${ }^{1,2}$ Of these, 174/231 had APOE genotyping. APOE genotyping began after the initial genetic portion of the study, so some subjects had died or were otherwise inaccessible for this determination. Nonetheless, APOE samples include all affected living subjects with any combination of FTD, IBM or PDB known to us. Of the 174 with $A P O E$ genotyping, 64 were affected (10 FTD, $58 \mathrm{IBM}, 30 \mathrm{PDB}$; the sum is $>64$ because of the overlap of these features in individuals). The remaining family members were asymptomatic carriers $(\mathrm{N}=25)$ or normal subjects without a VCP mutation $(\mathrm{N}=85)$. Basic characteristics of this $A P O E$ sample group are given in Table 1 . The
Table 1

Characteristics of 174 IBMPFD family members with Apolipoprotein E genotyping

\begin{tabular}{lccc}
\hline & Age (years) & Gender (F/M) & APOE 4 (\%) \\
\hline Affected & $51.3(10.2)$ & $36 / 28$ & 28.1 \\
FTD & $57.9(6.5)$ & $9 / 1$ & 70.0 \\
IBM & $52.3(9.9)$ & $32 / 26$ & 27.5 \\
PDB & $50.7(9.9)$ & $14 / 16$ & 26.6 \\
Asymptomatic & $37.3(8.0)$ & $12 / 13$ & 52.0 \\
No mutation & $47.3(15.3)$ & $50 / 35$ & 32.9 \\
\hline
\end{tabular}

F, female; M, male.

APOE4 (\%), the percentage of subjects with one or more APOE4 alleles \pm SD.

average age of affected family members in our study is considerably more that the average age of asymptomatic $V C P$ mutation carriers $(51.3 \pm 10.2$ SD years vs. $37.3 \pm 8.0, P<0.0001)$ as expected for an adult onset autosomal dominant disorder. Family members with no mutation $(47.3 \pm 15.3$ years $)$ did not differ in age from affected.

For the purposes of analysis, age is defined as either the current age or the age of death for each subject. Therefore, a deceased subject is treated in the analysis as the equivalent of a living person of the same age as the age of death. This is the clinically relevant age rather than the age the blood sample was drawn because APOE is a lifelong genetic trait uninfluenced by sample timing. The primary analysis consisted of two complementary logistic regressions (JMP 6.0, SAS Institute 2005, Cary, NC). The first regression (Table 2; 170 subjects) used FTD (presence or absence) to test associations of this dependent variable with age, gender, $A P O E 4$ allele, possession of at least one MAPT $\mathrm{H} 2$ haplotype, IBM (presence/absence), PDB (presence/absence), and APOE 4 family membership ( 1 if any

\section{Table 2}

Results of the logistic regression model fit in 170 IBMPFD family members to assess the association of FTD with apolipoprotein E4 genotype and MAPT $\mathrm{H} 2$ haplotype

\begin{tabular}{lccl}
\hline Variable & $\begin{array}{c}\text { Parameter estimate } \\
(95 \% \text { confidence limits })\end{array}$ & $\mathrm{L}-\mathrm{R} \chi^{2}$ & $p$-value \\
\hline APOE 4 & $2.01(0.85-3.73)$ & 13.9 & 0.0002 \\
MAP- $\tau$ H2 & $0.33(-0.56-1.28)$ & 0.5 & 0.5 \\
Age, years & $-0.13(-0.25-0.03)$ & 6.8 & 0.009 \\
Gender & $-1.07(-2.68-0.01)$ & 3.9 & 0.05 \\
Family APOE 4 & $-1.07(-2.77-0.29)$ & 2.4 & 0.1 \\
IBM & $1.86(0.71-3.67)$ & 11.9 & 0.0006 \\
PDB & $-0.07(-1.13-0.91)$ & 0.0 & 0.89 \\
\hline
\end{tabular}

APOE 4 was significant for the presence of dementia, but not MAPT H2 haplotype. Confidence limits (95\%) for the logistic parameter estimates are given in column two. Family $A P O E 4$ is a binary variable identifying members of pedigrees with at least one $A P O E 4$ genotype member. Older affected subjects are more likely to demonstrate dementia, an expected finding from clinical observation.

$\mathrm{L}-\mathrm{R}$, likelihood ratio. 
Table 3

Results of the logistic regression model fit in 174 IBMPFD family members to assess the association of APOE genotype with FTD, IBM, and PDB

\begin{tabular}{lccc}
\hline Variable & $\begin{array}{c}\text { Parameter estimate } \\
(95 \% \text { confidence limits })\end{array}$ & $\mathrm{L}-\mathrm{R} \chi^{2}$ & $p$-value \\
\hline FTD & $1.18(0.42-2.05)$ & 9.5 & 0.002 \\
IBM & $-0.06(-1.09-1.12)$ & 0.0 & 0.9 \\
PDB & $-0.05(-0.62-0.73)$ & 0.0 & 0.9 \\
Age & $0.01(-0.01-0.04)$ & 1.0 & 0.3 \\
Gender & $-0.03(-0.38-0.31)$ & 0.0 & 0.8 \\
Family FTD & $-0.09(-0.59-0.45)$ & 0.1 & 0.7 \\
Affected [affected] & $0.61(-0.91-2.34)$ & 0.6 & 0.5 \\
Affected [carrier] & $-0.64(-1.65-0.29)$ & 1.7 & 0.2 \\
\hline
\end{tabular}

$A P O E 4$ was significantly associated with the presence of FTD, but not with IBM or PDB. Confidence limits (95\%) for the logistic parameter estimates are given in column two. Family FTD is a binary variable identifying members of pedigrees with at least one FTD member. Affected refers to the genetic and clinical status of individuals classified into three levels: affected, asymptomatic mutation (carrier), and no mutation.

$\mathrm{L}-\mathrm{R}$, likelihood ratio.

family member within a pedigree had an $A P O E 4$ genotype, 0 otherwise). There were 170 MAPT haplotypes available out of 174 family members with $A P O E$ determinations; 111 had diplotype $\mathrm{H} 1 / \mathrm{H} 1,51 \mathrm{H} 1 / \mathrm{H} 2$ and $8 \mathrm{H} 2 / \mathrm{H} 2$. The second regression (Table $3 ; 174$ subjects) used the APOE 4 genotype (presence or absence) as the dependent variable, with age, gender, FTD (presence/absence), IBM (presence/absence), PDB (presence/ absence), genetic status (affected, asymptomatic mutation carrier, and no mutation), and FTD family membership (1 if any family member within a pedigree had FTD, 0 otherwise) as independent variables. This analysis was designed to demonstrate any significant associations with APOE 4 genotype while controlling for potential confounds. Likelihood ratio $\chi^{2}<0.05$ was chosen for testing significance.

When pedigree or $V C P$ mutation type was entered directly in the regressions by designating a unique identifier for each pedigree and mutation, the estimates became unstable, because among the 15 families carrying 6 mutations there were too many levels with too few members $(\mathrm{N}<10)$. For example the most common of the 6 mutations (resulting in an R155H substitution in $V C P$ ) was present in $8 / 15$ families, which include $136(80 \%)$ of family members. To capture the potential confound of family membership, binary family membership variables (dementia and $A P O E$ 4) were created instead, and used in the two regressions to adjust for chance associations between FTD and APOE 4 within particular families. The FTD family membership variable encoded 151 family members in 10 families with at least one FTD affected, and 23 members of 5 families without FTD. The APOE 4 family membership variable coded 154 family members in 10 families with at least one APOE 4 genotype member, and 20 members of 5 families without APOE 4. This procedure allowed reliable estimates.

To supplement the two primary analyses we used a one-way ANOVA with an $F$ test for significance with a $P=0.05$ thresh- old to examine specifically whether subjects with the APOE 4 genotype were older than those without it, a potential bias because the likelihood of FTD increases with age. Using the same method we also divided the subjects by genetic status (affected, asymptomatic carrier, and no mutation) to compare average age by $A P O E 4$ genotype (present/absent) for each status level.

\section{RESULTS}

The age of onset of dementia in the study subjects was median 52.4 years (IQR 11.5 years, range $42-62$ years). Even the oldest of the subjects was below 65 years, and the median age was well below that expected for $\mathrm{AD}$, supporting the validity of the FTD diagnosis. Autopsies obtained in three of the subjects demonstrated a characteristic IBMPFD pathology as reported by us recently. ${ }^{17}$ In family interviews we asked specifically about the character of the dementia, emphasizing the rate of progression and the language, behavioral and parkinsonian motor abnormalities. Therefore we feel the diagnosis of FTD was reliable and the likelihood of misclassification low.

The notable finding is that FTD is associated with APOE 4 genotype in IBMPFD $(P=0.0002$; Table 2$)$, but not with the $M A P T \mathrm{H} 2$ haplotype $(P=0.5)$. Increasing age was independently associated with FTD $(P=0.01)$. There was no difference in age between $A P O E 4$ carriers $(45.6 \pm 13.3$ SD years) and noncarriers $(48.3 \pm 13.5 \mathrm{SD}$ years; $P=0.22)$. There was a significant association of FTD with IBM, but not with PDB. There was no gender effect, and no effect of APOE 4 family membership (families with at least one member having one or more APOE 4 alleles).

The complementary analysis examined association between APOE 4 genotype, FTD, IBM and PDB (Table 3). Only FTD was associated with $A P O E 4$ genotype $(P=0.002)$, after adjustment for age, gender, membership in a pedigree with at least one FTD member, and genetic status (affected, asymptomatic mutation carrier, and no mutation).

There was no difference in average age by $A P O E 4$ genotype (present/absent) overall (50.1 \pm 7.5 vs. $51.8 \pm 11.1, P=0.56)$, or by genetic status: affected $(50.1 \pm 2.4$ vs. $51.8 \pm 1.5, P=$ $0.56)$, asymptomatic carrier $(36.7 \pm 10.6$ vs. $38.0 \pm 4.0 ; P=$ 0.70 ), and no mutation ( $46.9 \pm 15.4$ vs. $47.6 \pm 15.3, P=0.84$ ).

\section{DISCUSSION}

The finding of the relatively low frequency of FTD compared to that of IBM and PDB in affected IBMPFD mutation carriers led us to investigate potential modifying genes to account for this variability. We investigated $A P O E$ in this role because of its known effect in Alzheimer's disease, another form of neurodegenerative dementia. The subjects with FTD in our study had an early age of onset, and the behavioral, language and motor features typical of the disorder distinguishing FTD from Alzheimer's disease. We found that FTD was associated with APOE 4 genotype, but not with the MAPT 
$\mathrm{H} 2$ haplotype implicated in sporadic frontotemporal lobar degeneration.

The low frequency of FTD has potential explanations other than an APOE modifier effect. There is a potential for under diagnosis, as not all subjects in this study were seen by a qualified psychiatrist or neurologist. However, under diagnosis alone would not be expected to create the imbalance of cases of FTD in APOE 4 carriers we observed. Individuals might also succumb to other complications of the disorder prior to developing the dementia. Severe myopathic complications include fatal pneumonia due to neurogenic respiratory weakness, particularly at a late stage. The strong association of IBM with FTD observed in this study emphasizes this point. In these cases the imbalance of APOE 4 in observed FTD cases could be interpreted as a protective effect on the myopathy in IBMPFD. Such a protective effect appears unlikely, as it has no support in the literature regarding any tissue type, but the possibility nonetheless needs to be acknowledged. Alternatively, if APOE 4 reduced the age of onset of FTD, as has been observed in AD, dementia might be more frequently observed before these severe myopathic complications could occur. ${ }^{18}$ We do not currently have enough reliable data on FTD age of onset to test this hypothesis, but plan a survival analysis as more information is collected in future years.

Another challenge to interpreting these data for a hypothesized APOE modifier effect is that we cannot ascertain now, with certainty, whether a given individual who carries a $V C P$ mutation, and who is currently without FTD, might develop FTD some time in the future. Affected APOE 4 carriers without dementia are younger than APOE 4 carriers with FTD (44.2 \pm 13.4 SD vs. $55.7 \pm 5.2$ years; $\mathrm{p}$-value for this age difference, 0.03). Inclusion of these younger nondemented APOE 4 subjects would be expected to bias the results against the study hypothesis, but despite this possible bias, the APOE modifier effect was still observed. In contrast, there is no age difference between subjects with and without the APOE 4 allele, suggesting that the comparison between these groups is not biased by including older subjects with APOE 4 genotype who might be more likely to be demented. Adjusting for age and the other variables included in the analysis also helps address this issue. The statistical conclusion from our analysis is that the association of FTD with APOE 4 is unlikely due to chance even considering these adjustments. We are predicting on the basis of the currently available evidence that those mutation carriers with an APOE 4 genotype, regardless of whether they are currently affected, are more likely to develop FTD than those without $A P O E$ 4. Future data collection and analysis demonstrating a strengthening or a weakening of this observed association between FTD and APOE 4 remains to be seen.

Studies investigating $A P O E$ isoforms in more common forms of FTD have been conflicting. ${ }^{7,9,10,19,20}$ A meta-analysis of 10 case-control studies with a total of 364 FTD patients and 2,671 controls did not reach statistical significance for an association between APOE isoforms and FTD. Analysis of a subset of studies using only neuropathologically confirmed cases showed a significant increase in APOE 2 allele frequency in FTD. ${ }^{21,22} \mathrm{FTD}$ is heterogeneous both clinically and pathologically. ${ }^{23} \mathrm{An}$ association between the fluent aphasic clinical form of FTD (but not other clinical FTD forms) and APOE 4 was found by Short et al. ${ }^{24}$ Recently, the APOE 4 allele has been found to be overrepresented in clinical FTD within a homogenous population in southern Italy, ${ }^{14}$ and in neuropathologically-verified Pick's disease and dementia lacking distinctive histopathology (DLDH). ${ }^{25}$

Tau pathology is characteristic of frontotemporal dementia associated with mutations in the MAPT gene on chromosome 17 , and in a considerable proportion ( $40 \%$ in one series) of sporadic cases. ${ }^{26}$ However, many other cases of FTD, e.g. associated with motor neuron disease, are free of tau pathology, instead demonstrating ubiquitin inclusions. ${ }^{27}$ A recent study suggested that the APOE 4 allele was associated with the presence of Alzheimer's-like pathology in FTD. ${ }^{6}$ This suggests that potential modifier effects of $A P O E 4$ may depend on the strength of the linkage between specific pathogenic pathways involved in particular forms of FTD and possibly separate pathogenic pathways involving APOE.

Clinical subtypes of FTLD have different tau and APOE genotype frequencies, suggesting these genes may influence the clinical presentation. ${ }^{24}$ In 2006, Ghidoni et al. investigated the association between the MAPT haplotypes and sporadic (sFTD) or familial FTD (FFTD) and found an overrepresentation of $\mathrm{H} 2$ haplotype and of $\mathrm{H} 2 \mathrm{H} 2$ genotype in FFTD patients. This association was even stronger in APOE 4 negatives. ${ }^{11,12,14}$ In our study including IBMPFD subjects we found no association between MAPT $\mathrm{H} 2$ haplotype and an increase risk of developing dementia.

At present there is little data to demonstrate links between VCP and APOE pathogenic pathways in IBMPFD. There are many theoretical points of linkage, however. VCP is a clathrinbinding protein involved in endocytosis, Golgi processing, and membrane fusion, and is required for export of proteins from the endoplasmic reticulum to the cytosol.,328-32 VCP has also been recently described as a targeting factor for specific proteins, e.g. nuclear factor kappa-B, to the ubiquitin proteosome pathway. ${ }^{33}$ Because APOE is though to exert its pathologic effects through alterations in the processing of A- $\beta$ peptide, ${ }^{34}$ there are several potential points during uptake and intracellular processing of A- $\beta$ where mutated VCP could influence the APOE- Abeta interaction, or the interaction of APOE with another, as yet unknown pathogenic peptide. In the current study APOE 4 genotype was associated with FTD but not with IBM or PDB, suggesting that the putative VCP-APOE interaction may be specific to brain and not muscle or bone.

One caveat regarding our study is that the sample of affected subjects was heterogeneous with respect to the number of family members per pedigree and the representation of specific mutations. One specific base change, $464 \mathrm{G}>\mathrm{A}$ in exon 5, was present in $80 \%$ of mutation carriers. The number of family members per pedigree was marginally more uniform; nonetheless, 9/15 families had fewer than 10 enrolled members, the remaining 6 families accounting for $72 \%$ of the study subjects. This made it difficult to assess the role of specific mutations and FTD. The number of affected subjects with APOE deter- 
minations was relatively small, 64 , with 10 FTD subjects. Given the relatively rarity of IBMPFD, we are likely to supplement these data only slowly after several more years of study, when more $V C P$ mutation carriers develop clinical symptoms.

Nonetheless, this study of $A P O E$ genotype in families with VCP mutations suggests that there is an increased risk of FTD in IBMPFD subjects harboring the APOE 4 isoform. We hope these results stimulate further investigation of molecular links between $V C P$ and $A P O E$ that may lead to a better understanding of the key pathogenetic factors in the etiology of the three cardinal components of IBMPFD. Future pathologic examination of IBMPFD subjects will be particularly helpful in defining relative contributions of tau, ubiquitin, $V C P$ and amyloid brain pathologies in this form of FTD.

\section{ACKNOWLEDGMENTS}

We would like to thank the physicians who referred patients, and the families for their continued support. Funding of this study is from NIAMS, National Institutes of Health (R03 AR 46869, R01 AR050236), Muscular Dystrophy Association, Paget Foundation, and previously from the Excellence in Academic Medicine Program at Southern Illinois University School of Medicine and NINDS K02 NS02157 award.

\section{References}

1. Kovach MJ, Waggoner B, Leal SM, Gelber D, et al. Clinical delineation and localization to chromosome 9p13.3-p12 of a unique dominant disorder in four families: hereditary inclusion body myopathy, Paget disease of bone, and frontotemporal dementia. Mol Genet Metab 2001;74:458-475.

2. Watts GD, Wymer J, Kovach MJ, Mehta SG, et al. Inclusion body myopathy associated with Paget disease of bone and frontotemporal dementia is caused by mutant valosin-containing protein. Nat Genet 2004;36:377-381

3. Woodman PG. p97, a protein coping with multiple identities. J Cell Sci 2003;116: 4283-4290.

4. Saunders AM, Schmader K, Breitner JC, Benson MD, et al. Apolipoprotein E epsilon 4 allele distributions in late-onset Alzheimer's disease and in other amyloid-forming diseases. Lancet 1993;342:710-711.

5. Corder EH, Saunders AM, Strittmatter WJ, Schmechel DE, et al. Gene dose of apolipoprotein E type 4 allele and the risk of Alzheimer's disease in late onset families. Science 1993;261:921-923.

6. Josephs KA, Tsuboi Y, Cookson N, Watt H, et al. Apolipoprotein E epsilon 4 is a determinant for Alzheimer-type pathologic features in tauopathies, synucleinopathies, and frontotemporal degeneration. Arch Neurol 2004;61:1579-1584.

7. Engelborghs S, Dermaut B, Goeman J, Saerens J, et al. Prospective Belgian study of neurodegenerative and vascular dementia: APOE genotype effects. J Neurol Neurosurg Psychiatry 2003;74:1148-1151.

8. Rosso SM, van Swieten JC, Roks G. van Duijn CM, et al. Apolipoprotein E4 in the temporal variant of frontotemporal dementia. Journal of Neurology Neurosurgery and Psychiatry 2002;72:820.

9. Pickering-Brown SM, Owen F, Isaacs A, Snowden J, et al. Apolipoprotein E epsilon4 allele has no effect on age at onset or duration of disease in cases of frontotemporal dementia with pick- or microvacuolar-type histology. Exp Neurol 2000;163:452-456.

10. Geschwind D, Karrim J, Nelson SF, Miller B. The apolipoprotein E epsilon4 allele is not a significant risk factor for frontotemporal dementia. Ann Neurol 1998;44:134 138
11. Ghidoni R, Signorini S, Barbiero L, Sina E, et al. The H2 MAPT haplotype is associated with familial frontotemporal dementia. Neurobiol Dis 2006;22:357-362.

12. Borroni B, Yancopoulou D, Tsutsui M, Padovani A, et al. Association between tau H2 haplotype and age at onset in frontotemporal dementia. Arch Neurol 2005;62: 1419-1422.

13. Myers AJ, Kaleem M, Marlowe L, Pittman AM, et al. The H1c haplotype at the MAPT locus is associated with Alzheimer's disease. J Hum Mol Genet 2005;14:23992404.

14. Bernardi L, Maletta RG, Tomaino C, Smirne N, et al. The effects of APOE and tau gene variability on risk of frontotemporal dementia. Neurobiol Aging 2005;27:702709 .

15. Addya K, Wang YL, Leonard DG. Optimization of Apolipoprotein E Genotyping. Mol Diagn 1997;2:271-276.

16. Baker M, Litvan I, Houlden H, Adamson J, et al. Association of an extended haplotype in the tau gene with progressive supranuclear palsy. Hum Mol Genet 1999;8: 711-715.

17. Forman MS, Mackenzie IR, Cairns NJ, Swanson E, et al. Novel ubiquitin neuropathology in frontotemporal dementia with valosin-containing protein gene mutations. J Neuropathol Exp Neurol 2006;65:571-581.

18. Blacker D, Haines JL, Rodes L, Terwedow H, et al. APOE-4 and age at onset of Alzheimer's disease: the NIMH genetics initiative. Neurology 1997;48:139-147.

19. Gustafson L, Abrahamson M, Grubb A, Nilsson K, et al. Apolipoprotein-E genotyping in Alzheimer's disease and frontotemporal dementia. Dement Geriatr Cogn Disord 1997;8:240-243.

20. Fabre SF, Forsell C, Viitanen M, Sjogren M, et al. Clinic-based cases with frontotemporal dementia show increased cerebrospinal fluid tau and high apolipoprotein $\mathrm{E}$ epsilon4 frequency, but no tau gene mutations. Exp Neurol 2001;168:413-418.

21. Verpillat P, Camuzat A, Hannequin D, Thomas-Anterion C, et al. Apolipoprotein E gene in frontotemporal dementia: an association study and meta-analysis Association between the extended tau haplotype and frontotemporal dementia. Eur J Hum Genet 2002;10:399-405.

22. Verpillat P, Camuzat A, Hannequin D, Thomas-Anterion C, et al. Association between the extended tau haplotype and frontotemporal dementia. Arch Neurol 2002; 59:935-939.

23. Kertesz A, Hillis A, Munoz DG. Frontotemporal degeneration, Pick's disease, Pick complex, and Ravel. Ann Neurol 2003;54:S1-S2.

24. Short RA, Graff-Radford NR, Adamson J, Baker M, et al. Differences in tau and apolipoprotein E polymorphism frequencies in sporadic frontotemporal lobar degeneration syndromes. Arch Neurol 2002;59:611-615.

25. Mott RT, Dickson DW, Trojanowski JQ, Zhukareva V, et al. Neuropathologic, biochemical, and molecular characterization of the frontotemporal dementias. J Neuropathol Exp Neurol 2005;64:420-428.

26. Taniguchi S, McDonagh AM, Pickering-Brown SM, Umeda Y, et al. The neuropathology of frontotemporal lobar degeneration with respect to the cytological and biochemical characteristics of tau protein. Neuropathol Appl Neurobiol 2004;30: $1-18$.

27. Woulfe J, Kertesz A, Munoz DG. Frontotemporal dementia with ubiquitinated cytoplasmic and intranuclear inclusions. Acta Neuropathol (Berl) 2001;102:94-102.

28. Druck T, Gu Y, Prabhala G, Cannizzaro LA, et al. Chromosome localization of human genes for clathrin adaptor polypeptides AP2 beta and AP50 and the clathrinbinding protein, VCP. Genomics 1995;30:94-97.

29. Zhang SH, Liu J, Kobayashi R, Tonks NK. Identification of the cell cycle regulator VCP (p97/CDC48) as a substrate of the band 4.1-related protein-tyrosine phosphatase PTPH1. J Biol Chem 1999;274:17806-17812.

30. Ye Y, Meyer HH, Rapoport TA. The AAA ATPase Cdc48/p97 and its partners transport proteins from the ER into the cytosol. Nature 2001;414:652-656.

31. Pleasure IT, Black MM, Keen JH. Valosin-containing protein, VCP, is a ubiquitous clathrin-binding protein. Nature 1993;365:459-462.

32. Zalk R, Shoshan-Barmatz V. ATP-binding sites in brain p97/VCP (valosin-containing protein), a multifunctional AAA ATPase. Biochem J 2003;374:473-480.

33. Dai RM, Li CC. Valosin-containing protein is a multi-ubiquitin chain-targeting factor required in ubiquitin-proteasome degradation. Nat Cell Biol 2001;3:740-744.

34. Holtzman DM. In vivo effects of APOE and clusterin on amyloid-beta metabolism and neuropathology. J Mol Neurosci 2004;23:247-254 